\title{
Conocimiento de vocabulario, representaciones fonológicas y sensibilidad fonológica en niños pequeños de distinto sector social de procedencia
}

\section{Vocabulary knowledge, phonological representations and phonological sensitivity in Spanish-speaking low-and middle-SES preschoolers}

\author{
Beatriz Diuk $^{1 *}$, Ana María Borzone y Rubén Ledesma** \\ Consejo Nacional de Investigaciones Científicas y Técnicas \\ *Universidad Nacional de San Martín, Argentina \\ **Universidad de Mar del Plata, Argentina
}

(Recepción: Diciembre 2009 - Aceptación: Junio 2010)

\begin{abstract}
Resumen
En el presente trabajo se analizaron las relaciones entre una serie de habilidades consideradas precursoras de la lectura y la escritura convencional en 80 niños de 4 años de edad, diferenciados por sector social de procedencia. Se evaluó a los niños con tareas de vocabulario receptivo y productivo, representaciones fonológicas y reconocimiento de rima y de sílaba inicial. Se obtuvieron diferencias estadísticamente significativas entre grupos en todas las tareas exceptuando la identificación de sílabas. Los análisis de regresión mostraron un patrón similar entre grupos: el vocabulario receptivo predijo la identificación de rimas y la tarea que evalúa representaciones fonológicas predijo el reconocimiento de sílabas. La diferencia entre los predictores de una y otra habilidad sugiere que el desarrollo léxico temprano proporciona el marco para el desarrollo de niveles rudimentarios de sensibilidad fonológica, pero la identificación de unidades menores requiere de representaciones fonológicas más especificadas.

Palabras claves: representaciones fonológicas, sensibilidad fonológica, conocimiento de vocabulario, diferencias socioeconómicas.
\end{abstract}

\begin{abstract}
The aim of this study was to examine the relationships among vocabulary knowledge, phonological representations and phonological sensitivity in 80 Spanish-speaking preschool children from middle- and low-SES families. Significant social class differences were obtained on all tasks except syllable matching. Regression analyses were carried out to test the predictive power of vocabulary knowledge and accuracy of phonological representations on the phonological sensitivity measures. Receptive vocabulary predicted rhyme identification. Syllable matching was predicted by a task tapping accuracy of phonological representations. The fact that rhyme identification was predicted by vocabulary knowledge but syllable matching was predicted by a measure tapping accuracy of phonological representations in both groups suggests that early lexical development sets the stage for the development of the lower levels of phonological sensitivity but identification of smaller units requires more accurate and segmented phonological representations.

Key words: phonological representations, phonological sensitivity, vocabulary knowledge, socioeconomic differences.
\end{abstract}

1 Correspondencia a: Beatriz Diuk. Tte. Gral Perón 2158 (C1040AAH), Buenos Aires, Argentina. Teléfono: 054-011-49531477. Email: beadiuk@gmail.com. 
Las diferencias entre sectores sociales en habilidades lectoras han sido ampliamente documentadas. Los niños de nivel socioeconómico (NSE) bajo tienden a experimentar dificultades en una proporción mayor que los niños de otros sectores sociales (Borzone \& Diuk, 2001; Diuk, 2007; Diuk \& Borzone, 2006; Diuk, Signorini \& Borzone; Bowey, 1995; Hecht, Burgess, Torgesen, Wagner, \& Rashotte, 2000; Nicholson, 1997). Estas diferencias resultan particularmente relevantes cuando se compara a niños que viven en condiciones de pobreza extrema con niños de NSE medio (Chatterji, 2006). En América Latina, la prevalencia de las dificultades en niños de comunidades desaventajadas ha sido estimada en el 25\% (Capovilla \& Capovilla, 2004), una proporción que excede las estimaciones de las dificultades lectoras en la sociedad global, ubicadas entre el 5\% y el 15\% (Snow, Burns, \& Griffin, 1998). Dado que el 33\% de la población de América Latina vive en condiciones de pobreza (CEPAL, 2009), resulta necesario comprender la naturaleza de las dificultades experimentadas por los niños que crecen en estos contextos.

Un modo de contribuir a esta comprensión es procurar identificar en qué habilidades asociadas a la lectura se producen diferencias tempranas entre los niños de distintos sectores sociales. Estudios previos han identificado diferencias estadísticamente significativas en el desarrollo de la sensibilidad fonológica, esto es, la habilidad de identificar y manipular sonidos del lenguaje (Borzone, 1997). La sensibilidad fonológica es uno de los principales predictores de la facilidad con la que los niños aprenden a leer (ver Ehri, Nunes, Willows, Schuster, Yaghoub-Zadeh, \& Shanahan, 2001).

En el presente estudio se busca ir más allá de la identificación de diferencias en sensibilidad fonológica y analizar el desarrollo de las habilidades cognitivas que se consideran precursoras del desarrollo de la conciencia fonológica.

De hecho, la importancia de la sensibilidad fonológica en la adquisición lectora ha llevado a muchos investigadores a avanzar en la búsqueda de diferencias individuales en las variables que subyacen a esta habilidad. Se ha hipotetizado que el desarrollo de la conciencia fonológica es altamente dependiente del estatus de las representaciones fonológicas de las palabras en el léxico mental (Fowler, 1991). El desempeño de los niños en las tareas de conciencia fonológica puede reflejar la precisión y el nivel de segmentación de las representaciones fonológicas de las palabras que el niño debe analizar. En efecto, en estas tareas, los niños deben realizar una serie de operaciones sobre las representaciones del lenguaje oral y parece razonable suponer que representaciones más precisas serán más sencillas de manipular.

Por otra parte, varios investigadores han propuesto que la calidad de las representaciones de ítems léxicos se relaciona con el conocimiento de vocabulario. La presión producida por el incremento en el número de ítems en el léxico mental daría lugar a una estructuración más segmental de las representaciones (Metsala, 1999; Metsala \& Walley, 1998). Ahora bien, la evidencia acerca de la relación entre estas habilidades no es aún consistente $\mathrm{y}$, en particular, ha sido escasamente explorada en niños de distintos sectores sociales.

En consecuencia, el presente estudio tiene por objeto explorar la relación entre el vocabulario, las representaciones fonológicas y la sensibilidad fonológica en niños diferenciados por sector social de procedencia: niños de familias de NSE medio y niños que crecen en contextos de pobreza. Estudios previos sugieren que los niños de NSE bajo tienden a presentar niveles de desempeño inferiores a los de niños de otros sectores sociales (Borzone, 1997; Lonigan, Burgess, Anthony \& Barker, 1998). El interés de este trabajo fue ir más allá de la comparación entre niveles de desempeño para explorar las relaciones entre variables a fin de analizar si el patrón de asociación es similar entre grupos. Un patrón de correlaciones similares sugeriría que los niños de NSE bajo están desarrollando las mismas habilidades a un ritmo más lento, y no con un patrón cualitativamente diferente 


\section{Vocabulario, representaciones fonológicas y sensibilidad fonológica en estudios previos}

La relación entre el tamaño del vocabulario y la lectura ha sido ampliamente estudiada y existe creciente evidencia de que las habilidades de vocabulario oral no están asociadas al desarrollo de la lectura de palabras en hablantes nativos (Muter, Hulme, Snowling, \& Stevenson, 2004; Ricketts, Nation, \& Bishop, 2007) ni en niños que aprenden a leer en una segunda lengua (Araujo, 2002; August \& Shanahan, 2006; Chiappe, Siegel, \& Gottardo, 2002).

Sin embargo, el vocabulario podría ejercer una influencia a edad más temprana al afectar el desarrollo de habilidades asociadas a la lectura tales como la calidad de las representaciones fonológicas o la sensibilidad fonológica. Sin embargo, la relación entre estas habilidades no es clara. Algunos estudios han encontrado evidencia de una relación entre vocabulario y conciencia fonológica (Chaney, 1994; Metsala, 1999), en tanto en otros no estuvieron asociados (Garlock, Walley, \& Metsala, 2001). Lonigan et al. (1998) encontraron que el vocabulario y la sensibilidad fonológica estaban correlacionados en su muestra de niños de 4 y 5 años de edad de NSE medio pero no en el grupo de NSE bajo.

Asimismo, la evidencia acerca de la relación entre la precisión de las representaciones fonológicas y la sensibilidad fonológica es inconsistente, dado que algunos estudios han encontrado asociaciones entre estas variables (Elbro, Borstrom \& Klint Petersen, 1998; McBride-Chang, Wagner, \& Chang, 1997; Swan \& Goswami, 1997) pero otros no (Wesseling \& Reitsma, 2001). De hecho, se han encontrado patrones diferentes en función de las tareas utilizadas para medir las representaciones fonológicas y del tamaño de la unidad lingüística empleada en la tarea de sensibilidad fonológica. La mayor parte de los estudios encontraron una relación entre la calidad de las representaciones fonológicas y la conciencia fonémica, es decir, la habilidad para manipular fonemas. (Elbro et al., 1998; Roberts, 2005; Thomas \& Sénéchal, 2004).

Sin embargo, la evidencia no es tan consistente cuando se opera con otras unidades lingüísticas. Carroll, Snowling, Hulme y Stevenson (2003) encontraron que las habilidades articulatorias de los niños prescolares predecían la conciencia fonémica un año más tarde, pero la tarea articulatoria no presentó correlaciones con la prueba de rima en ninguna de las tres evaluaciones realizadas en su estudio longitudinal. Swan y Goswami (1997) analizaron el desempeño de niños disléxicos en tareas de sensibilidad fonológica utilizando palabras para las cuales los niños tenían representaciones fonológicas precisas vs. palabras para las cuales las representaciones eran imprecisas. La calidad de las representaciones fonológicas afectó el desempeño en las pruebas que involucraban la manipulación de sílabas y ataque y rima, dado que los niños se desempeñaron mejor en las palabras para las cuales contaban con buenas representaciones fonológicas. En la prueba de sensibilidad fonémica, los niños presentaron un déficit también en las palabras cuyas representaciones eran precisas, sugiriendo que los niños presentaban representaciones fonológicas suficientemente adecuadas para el análisis en sílabas o rimas, pero no para un análisis fonémico.

En efecto, existe un creciente consenso en los estudios del desarrollo fonológico acerca de que las representaciones fonológicas no se encuentran, inicialmente, organizadas en torno a sonidos individuales o segmentos fonéticos. Es sólo gradualmente que las representaciones son reestructuradas segmentalmente. Estudios sobre la producción del habla infantil (Borzone \& Massone, 1979, 1985; Ferguson \& Farwell, 1975) proporcionaron evidencia empírica acerca del papel de la palabra como unidad de contraste en el habla temprana, dada la variación encontrada en la producción de una misma palabra. El desarrollo del habla infantil es considerado un proceso de diferenciación de unidades menores a partir de otras mayores. El niño no construye las palabras a partir de fonemas: los fonemas emergen de las palabras.

Se considera que los cambios evolutivos en la estructura de las representaciones fonológicas son impulsados por el crecimiento del vocabulario. Las nuevas palabras cuyas propiedades acústicas se superponen con palabras conocidas demandan, para su identificación, de una mayor segmentalidad en las representaciones (Metsala \& Walley, 1998). 
En síntesis, un mayor tamaño de vocabulario estaría asociado a representaciones fonológicas más especificadas y esta mayor precisión en las representaciones se asociaría a un mejor desempeño en pruebas de sensibilidad fonológica.

En el presente trabajo se buscó explorar esta relación en niños de 4 años de edad de NSE medio y bajo. Dado que se trata de niños pequeños, y en base a la evidencia acerca del menor desarrollo en la sensibilidad fonológica en niños de NSE bajo, resultó fundamental encontrar tareas evolutivamente apropiadas. En el caso de los niños pequeños con frecuencia se obtienen desempeños muy bajos en las tareas que evalúan conciencia fonémica (Borzone, 1997; Lonigan, et al., 1998; Signorini \& Borzone, 1996). Hindson, Byrne, Fielding-Barnsley, Newman, Hine, \& Shankweiler (2005) encontraron niveles limitados de conciencia fonémica en niños preescolares de NSE medio y sugirieron que la identificación de rimas podría reflejar más adecuadamente el nivel de sensibilidad fonológica de los niños de esta edad.

En el presente estudio se evaluó la sensibilidad fonológica utilizando pruebas de reconocimiento de rimas y sílabas, que pueden ser consideradas tareas que miden un nivel rudimentario de sensibilidad que se puede adquirir a edad temprana. Dado que la sensibilidad a niveles bajos y altos de complejidad lingüística son considerados partes de un continuo (Lonigan, Burgess, \& Anthony, 2000), contribuir a la comprensión del desarrollo de la sensibilidad a las rimas y a las sílabas puede aportar a la comprensión del proceso de adquisición lectora y al estudio de las diferencias socioeconómicas en estas habilidades.

\section{Método}

\section{Participantes}

Participaron de este estudio 80 niños: 41 niños de NSE bajo (24 niñas y 17 niños) y 39 niños (20 niñas y 19 niñas) de NSE medio. Los niños de NSE bajo asistían a un centro de educación inicial ubicado en una comunidad muy pobre de Buenos Aires, Argentina. La edad de los niños presentaba un rango de 52 a 63 meses $(M=59.38, S D=3.53)$. Los niños de NSE medio asistían a dos escuelas privadas de Buenos Aires. Sus edades estaban comprendidas entre los 53 y los 63 meses $(M=57.55$, $S D=3.62$ ).

Se obtuvieron datos sobre el nivel educativo de la madre y el padre y sobre el estatus ocupacional del adulto a cargo de cada niño (ver tabla 1). La ocupación de los adultos responsables fue establecida en base a los informes de los propios adultos y categorizada mediante la escala ocupacional desarrollada por Sautú (1992). La escala considera 9 categorías. Con una única excepción, no se produjeron solapamientos en las ocupaciones adultas entre los niños de los distintos grupos sociales. Los adultos del grupo de NSE bajo tenían ocupaciones ubicadas en las cuatro categorías inferiores de la escala en tanto los adultos del grupo de NSE medio presentaban trabajos correspondientes a las categorías 5 a 9. El modo para el grupo de NSE bajo fue 3, para el de NSE medio fue 8. Cuando se consideró la educación de los padres, se encontraron diferencias estadísticamente significativas en el número de años de educación entre grupos.

Cada centro educativo solicitó y obtuvo autorización de los padres para la participación en este estudio. 
Tabla 1: Información demográfica de ambos grupos de niños

\begin{tabular}{lccccc}
\hline \multirow{2}{*}{ Variable } & \multicolumn{2}{c}{ NSE bajo } & \multicolumn{2}{c}{ NSE medio } & \multirow{2}{*}{$t$} \\
& $M$ & $S D$ & $M$ & $S D$ & \\
\hline Educación de la madre & 7.69 & 1.84 & 13.19 & 1.88 & $10.685^{* *}$ \\
Educación del padre & 8.51 & 2.47 & 14.00 & 2.45 & $7.987^{* *}$ \\
Ocupación del adulto responsable & 3.12 & 0.65 & 6.94 & 1.41 & $14.973^{* *}$ \\
\hline
\end{tabular}

$* * p<.001$

\section{Medidas}

\section{Pruebas de sensibilidad fonológica}

Se administraron dos pruebas de sensibilidad fonológica: una prueba de identificación de rimas y una prueba de identificación de sílaba inicial. Con el objeto de controlar la frecuencia de las palabras, se seleccionó un grupo de palabras del diccionario de Alameda y Cuetos (1995). Se solicitó a seis maestras del centro educativo al que asistían los niños de NSE bajo que clasificaran las palabras como muy frecuentes, frecuentes o poco frecuentes para los niños. Solo palabras seleccionadas como frecuentes y muy frecuentes por las maestras fueron utilizadas. En un estudio piloto, los dibujos realizados para ilustrar las pruebas fueron presentados a 15 niños y se les solicitó que señalaran las palabras nombradas por el investigador. Solamente se incluyeron las palabras identificadas por todos los niños.

Prueba de identificación de rimas: la prueba consistía en tres ensayos y diez ítems de prueba. El experimentador presentaba a los niños una hoja con un dibujo en la parte superior y dos dibujos debajo. Se pronunciaba el nombre del primer dibujo y se solicitaba al niño que identificara cuál de los dibujos representados debajo rimaba con el primero. Se asignaba un punto por cada respuesta correcta.

Identificación de sílaba inicial: se trata de una prueba con el mismo formato.

\section{Pruebas de vocabulario}

Vocabulario receptivo: se administró el Test de Vocabulario en Imágenes Peabody (Dunn, Lugo, Padilla, \& Dunn, 1986)

Vocabulario expresivo: se utilizó el Test figura/palabra del vocabulario expresivo (Gardner, 1987).

\section{Prueba de representaciones fonológicas}

Prueba de corrección de la pronunciación: se adaptó al español la prueba de Fowler y Swainson (2004). En esta prueba, los niños oían un estímulo grabado (una palabra mal pronunciada) a través de un grabador escondido dentro de un títere. Se explicó a los niños que el títere estaba aprendiendo a hablar, pero que cometía muchos errores y necesitaba ayuda. Se solicitaba a los niños que repitieran la palabra errónea que habían escuchado. Luego se les presentaban tres dibujos de objetos semánticamente relacionados y los niños debían identificar la palabra intentada por el títere. Finalmente, se les pedía que pronunciaran la palabra correctamente tres veces. Se asignaron puntajes separados para la repetición de la palabra mal pronunciada, la identificación de la palabra intentada 
y la corrección de la pronunciación. Se asignó un punto a cada respuesta correcta. En el caso de la corrección de la pronunciación se obtuvieron dos puntajes: uno para la corrección en el primer intento y uno para la corrección en intentos sucesivos.

La tarea consistía en 20 palabras controladas por frecuencia y largo. La frecuencia fue establecida en base al diccionario de Alameda y Cuetos (1995) y al informe de las docentes. Las palabras cortas tenían dos sílabas y las palabras largas, tres o cuatro. Las pronunciaciones erróneas fueron derivadas de la palabra target a través de la elisión, adición, inversión o sustitución de fonemas.

\section{Procedimiento}

Los niños fueron evaluados en forma individual en un espacio silencioso en cada institución educativa. Cada niño participó de dos sesiones de aproximadamente 15 minutos cada una. Las sesiones tuvieron lugar en semanas sucesivas. Las dos pruebas de sensibilidad fonológica fueron aplicadas en sesiones diferentes. Las pruebas fueron administradas por la primera autora de este trabajo y por dos psicopedagogas especialmente entrenadas para la tarea.

\section{Resultados}

El análisis preliminar de los datos indicó que la prueba de identificación de rimas presentaba un desvío de la normalidad en ambos grupos. La transformación de esta variable mejoró la distribución pero no cambió el patrón de correlaciones entre variables ni el patrón de resultados de los análisis de regresión. En consecuencia, todos los análisis fueron realizados utilizando las variables no transformadas.

Los estadísticos descriptivos para ambos grupos se presentan en la tabla 2. Siguiendo a Lonigan et al. (1998), dadas las diferencias entre sectores sociales en las pruebas de sensibilidad fonológica y vocabulario, los análisis de consistencia interna fueron llevados a cabo por separado para las muestras de NSE medio y bajo (ver Tabla 2). Con el objeto de explorar la validez de las medidas de sensibilidad fonológica se condujo un análisis factorial exploratorio que mostró que la comunalidad era superior al $69 \%$. La carga factorial en ambos tests fue de .83 .

Tabla 2: Estadísticos descriptivos para todas las tareas por grupo

\begin{tabular}{|c|c|c|c|c|c|c|}
\hline & \multicolumn{2}{|c|}{ NSE bajo } & \multirow[b]{2}{*}{$\alpha$} & \multicolumn{2}{|c|}{ NSE medio } & \multirow[b]{2}{*}{$\alpha$} \\
\hline & M & SD & & M & SD & \\
\hline Identificación rima & 7.00 & 2.66 & .78 & 8.71 & 1.94 & .78 \\
\hline Identificación sílaba & 6.56 & 2.11 & .57 & 7.28 & 2.05 & .61 \\
\hline $\begin{array}{l}\text { Vocabulario receptivo (Puntaje } \\
\text { bruto) }\end{array}$ & 31.77 & 13.38 & - & 50.84 & 18.47 & - \\
\hline $\begin{array}{l}\text { Vocabulario expresivo (Puntaje } \\
\text { bruto) }\end{array}$ & 40.13 & 8.48 & - & 80.95 & 9.54 & - \\
\hline $\mathrm{CP}$ - imitación & 10.62 & 2.77 & .45 & 11.42 & 3.85 & .74 \\
\hline CP- identificación & 15.30 & 2.55 & .54 & 15.92 & 1.76 & .45 \\
\hline $\mathrm{CP}-$ corrección & 9.82 & 4.05 & .65 & 12.68 & 3.97 & .71 \\
\hline
\end{tabular}

Note: $\mathrm{CP}$ - prueba de corrección de la pronunciación

A continuación se analizaron las diferencias entre grupos. Se condujo un ANOVA multivariado con todas las medidas (identificación de rima y sílaba, prueba de representaciones fonológicas imitación, identificación y corrección y vocabulario receptivo y expresivo) con grupo (NSE medio 
vs. bajo) como factor intersujetos. El modelo fue significativo $\left(F(7,66)=6.504, p<.001, \eta^{2}=.408\right)$. Una serie de ANOVAS utilizando el ajuste de Bonferroni para comparaciones múltiples reveló diferencias significativas entre grupos para ambas medidas de vocabulario $(F(1,66)=31.421, p<$ $.001, \eta^{2}=.304, M S E=79.619$ para vocabulario expresivo y $F(1,66)=27.990, p<.001, \eta^{2}=.280$, $M S E=267.080$ para vocabulario receptivo), para la prueba de identificación de rimas $(F(1,66)=$ $\left.7.790, p<.01, \eta^{2}=.098, M S E=5.582\right)$ pero no para la prueba de identificación de sílabas $(F(1,66)$ $\left.=2.631, p=.08, \eta^{2}=.035, M S E=4.064\right)$.

En la prueba de corrección de la pronunciación no se encontraron diferencias significativas entre grupos en la imitación de la pronunciación del títere $\left(F(1,66)=1.233, p=.300, \eta^{2}=.017, M S E=\right.$ $10.704) \mathrm{ni}$ en el reconocimiento del objeto nombrado $\left(F(1,66)=1.470, p=.218, \eta^{2}=.020, M S E=\right.$ 4.993). Pero el grupo de NSE medio se desempeñó mejor al proporcionar la pronunciación correcta de la palabra. $\left(F(1,66)=11.046, p=.001 \eta^{2}=.133, M S E=15.536\right)$. Cabe señalar que solamente se consideró el puntaje obtenido en el primer intento de corregir la pronunciación, dado que sólo en 6 ocasiones (de 1600) los niños autocorrigieron una pronunciación inicialmente incorrecta.

Dado que se encontraron diferencias estadísticamente significativas entre grupos en la prueba de identificación de rimas pero no de sílabas, se decidió explorar la dificultad relativa de las pruebas al interior de cada grupo. Se llevaron a cabo dos ANOVAS de medidas repetidas con sensibilidad fonológica (rima y sílaba) como medida intra-sujetos. A pesar de que en ambos grupos la identificación de rimas presentó puntajes más altos que la identificación de sílabas, solo se encontraron diferencias estadísticamente significativas en el grupo de NSE medio $(F(1,38)=14.797, p<.001, M S E=2.434$, para el grupo de NSE medio y $F(1,39)=1.060, p=.309, M S E=3.819$, para el grupo de NSE bajo).

Las correlaciones entre las medidas para cada grupo se presentan en la Tabla 3. La mayor parte de las medidas presentaron correlaciones moderadas. Se encontraron diferencias entre grupos en las pruebas de vocabulario expresivo y de identificación de rimas, que no estuvieron correlacionadas en el grupo de NSE bajo. El patrón de correlaciones para los componentes de repetición e identificación en la prueba de representaciones fonológicas también resultó algo diferente entre grupos.

Tabla 3: Correlaciones entre tareas

\begin{tabular}{|c|c|c|c|c|c|c|c|}
\hline & $\begin{array}{l}\text { Id. } \\
\text { rima }\end{array}$ & $\begin{array}{c}\text { Id. } \\
\text { sílaba. }\end{array}$ & $\begin{array}{l}\text { Correcci } \\
\text { Imitación. }\end{array}$ & $\begin{array}{l}\text { ón Pronur } \\
\text { Identific }\end{array}$ & $\begin{array}{l}\text { ciación } \\
\text { Correcc. }\end{array}$ & $\begin{array}{l}\text { Voc. } \\
\text { Recep. }\end{array}$ & $\begin{array}{c}\text { Voc. } \\
\text { Expresivo }\end{array}$ \\
\hline Identificación rima & - & $.34 *$ & $.45^{* *}$ & .17 & $.39 * *$ & $.47 * *$ & .17 \\
\hline Identificación sílaba & $.36^{*}$ & - & .24 & .19 & $.45^{* *}$ & $.43 * *$ & $.43^{* *}$ \\
\hline CP - Imitación & $.39^{*}$ & .28 & - & .29 & $.483 * *$ & .133 & .161 \\
\hline CP Identificación. & .21 & .06 & $.51 * *$ & - & $.575 * *$ & .250 & $.514 * *$ \\
\hline $\mathrm{CP}$ - corrección & $.50 * *$ & $.43 * *$ & $.74 * *$ & $.59 * *$ & - & $53 * *$ & $.49 * *$ \\
\hline $\begin{array}{l}\text { Vocabulario } \\
\text { receptivo }\end{array}$ & $.54 * *$ & $.34 *$ & $.48 * *$ & .29 & $.48 * *$ & - & $.42 * *$ \\
\hline $\begin{array}{l}\text { Vocabulario } \\
\text { expresivo }\end{array}$ & $.46^{* *}$ & $.41 *$ & $.42 * *$ & $.42 * *$ & $.67 * *$ & $.56^{* *}$ & - \\
\hline
\end{tabular}

$* \mathrm{p}<.05, * * \mathrm{p}<.01$

Las correlaciones por encima de la diagonal corresponden al grupo de NSE bajo y las correlaciones por debajo de la diagonal, al grupo de NSE medio 
Con el objeto de examinar la relación entre el vocabulario y la tarea de representaciones fonológicas con las pruebas de sensibilidad fonológica, se llevaron a cabo análisis de regresión por pasos para cada grupo. En una primera regresión se tomó el puntaje en la prueba de identificación de rimas como variable dependiente y se introdujeron como variables predictoras los puntajes en las pruebas de vocabulario expresivo y receptivo y en la imitación, identificación y corrección de la pronunciación errónea. Como se muestra en la Tabla 4, el patrón de resultados fue el mismo para ambos grupos, con vocabulario receptivo como variable predictora. Esta variable explicó el $47 \%$ de la varianza en el grupo de NSE bajo y el $29 \%$ en el de NSE medio.

Otra serie de regresiones por pasos sucesivos fue llevada a cabo con identificación de sílaba como variable dependiente y vocabulario expresivo y productivo y los puntajes de imitación, identificación y corrección de la pronunciación errónea como variables predictoras (ver Tabla 5). Nuevamente, el patrón de resultados fue el mismo para ambos grupos, con corrección de la pronunciación como variable predictora. Esta variable explicó el 18\% y el 25\% de la varianza en los grupos de NSE bajo y medio, respectivamente.

Tabla 4: Análisis de regresión por pasos de las variables que predicen el reconocimiento de rimas

\begin{tabular}{ccccc}
\hline & $\beta$ & $\mathrm{R}^{2}$ & $\Delta \mathrm{R}^{2}$ & Probabilidad \\
\hline NSE bajo & .692 & .479 &, 464 & $<.001$ \\
NSE medio & .559 & .312 &, 291 & $<.001$ \\
\hline
\end{tabular}

Variable predictora: vocabulario receptivo

Tabla 5: Análisis de regresión por pasos de las variables que predicen el reconocimiento de sílabas

\begin{tabular}{ccccc}
\hline & $\beta$ & $\mathrm{R}^{2}$ & $\Delta \mathrm{R}^{2}$ & Probabilidad \\
\hline NSE bajo & .448 & .201 & .178 & $<.01$ \\
NSE medio & .517 & .268 & .245 & $<.01$ \\
\hline
\end{tabular}

Variable predictora: corrección de pronunciación

Dada la relevancia de los resultados obtenidos en la prueba de corrección de la pronunciación errónea, se llevó a cabo un análisis en detalle de los errores producidos por los niños en sus intentos por proporcionar la pronunciación correcta. Solamente los casos en los cuales los niños habían identificado correctamente el objeto a nombrar fueron considerados. Este análisis mostró que los niños produjeron cuatro tipos de errores: repetición de la pronunciación errónea del títere (el títere había dicho globlo por globo y el niño lo repetía, a pesar de haber reconocido el dibujo del globo), pronunciar una no palabra diferente de la nombrada por el títere (goblo, por ejemplo), producir una palabra fonológicamente similar a la intentada (lobo), o una palabra semánticamente relacionada con el estímulo (bola). 
Tabla 6: Porcentaje promedio de tipos de errors en la corrección de la pronunciación erróne $a$

\begin{tabular}{lccc}
\hline Tipo de error & NSE medio & NSE bajo & Mann-Whitney's U \\
\hline Repetición del input & 2.44 & 5.5 & $558.5 *$ \\
$\begin{array}{l}\text { No palabra diferente del input } \\
\text { Palabra semánticamente }\end{array}$ & 6.28 & 9.12 & 640.5 \\
$\begin{array}{l}\text { relacionada con el target } \\
\begin{array}{l}\text { Palabra fonológicamente } \\
\text { relacionada con el target }\end{array}\end{array}$ & 1.92 & 5.37 & 669 \\
\hline
\end{tabular}

Como se ve en la Tabla 6, los niños de NSE bajo produjeron más errores en todas las categorías pero solamente se encontraron diferencias estadísticamente significativas entre grupos en el tipo de error consistente en repetir la pronunciación del títere en lugar de proporcionar la forma correcta de la palabra $(U=558.5, p<.01)$. Cabe señalar que ningún niño produjo exclusivamente este tipo de respuesta, indicando que había comprendido la tarea pero que, en algunos estímulos, no lograba proporcionar la forma correcta de la palabra.

\section{Discusión}

Este trabajo tuvo por objeto analizar el patrón de relaciones entre el desempeño en pruebas de sensibilidad fonológica y variables que son consideradas precursores evolutivos de la sensibilidad fonológica - el conocimiento de vocabulario y la calidad de las representaciones fonológicas en el léxico mental. Estas relaciones fueron analizadas en niños de distinto sector social de procedencia a fin de explorar si los perfiles cognitivos de los niños pequeños de NSE medio y bajo eran similares.

En el grupo de NSE medio la identificación de rimas resultó más fácil que la detección de sílabas. Estos resultados convergen con los obtenidos por Cardoso-Martins (1995) para el portugués. Dado que tanto en español como en portugués la mayor parte de las palabras que riman presentan un solapamiento acústico suprasilábico, este resultado es esperable ya que los niños pueden apoyarse en información distribuida en la palabra (Murphy et al., 1989). En este sentido, Cardoso-Martins (1994) encontró que la habilidad para detectar rimas en portugués no parece requerir de la habilidad para identificar con precisión el segmento compartido por las palabras que riman, sino que involucraría una sensibilidad global a la similitud fonológica. En cambio, en la identificación de sílabas es necesario deslindar los segmentos a analizar.

Ahora bien, en el grupo de NSE bajo no se encontraron diferencias significativas entre el desempeño en la prueba de identificación rimas y en la de reconocimiento de sílabas. Adicionalmente, en base a investigaciones previas esperábamos que los niños de NSE medio alcanzaran un mejor desempeño que los de NSE bajo en todas las tareas (Adams, 1990; Borzone, 1997; Bowey, 1995; Hecht \& Greenfield, 2001; Lonigan et al., 1998; Raz \& Bryant, 1990). Esta predicción se verificó en el caso de las pruebas de vocabulario, en la prueba de representaciones fonológicas y en la de detección de rimas pero no en la de identificación de sílabas, en la que no se encontraron diferencias significativas entre grupos. Es decir, los niños de NSE bajo tuvieron mayores dificultades que los niños de NSE medio en la tarea más sencilla (reconocimiento de rimas) pero alcanzaron un nivel similar en la más compleja (detección de sílabas). Se trata de un resultado difícil de explicar.

Creemos, sin embargo, que variables instruccionales pueden haber producido este patrón de resultados. En efecto, las maestros del grupo de NSE bajo informaron que las actividades de identificación de sílabas formaban parte de su curriculum habitual, no así la identificación de rimas. Ninguno de los maestros de los grupos de NSE medio informó una estrategia instruccional similar. En ocasiones incluían canciones, rimas y juegos con sonidos en las actividades de aula, pero no 
había un trabajo sistemático de análisis fonológico de las palabras. En el grupo de NSE bajo, en cambio, las actividades destinadas a promover la identificación de sílabas tenían lugar dos o tres veces a la semana.

La incidencia de la intervención pedagógica en el desarrollo de la sensibilidad fonológica está ampliamente documentada. Estudios de entrenamiento en conciencia fonológica con pequeños grupos de niños han mostrado que es posible mejorar los niveles de sensibilidad fonológica de los niños a través de la intervención sistemática (Borzone, 1997; Bradley \& Bryant, 1883; Bus \& van Ijzendoorn, 1999; Defior \& Tudela, 1994; Ehri et al., 2001; Hecht \& Close, 2002; Signorini \& Borzone, 1996). Asimismo, existe evidencia creciente de que las intervenciones con grupos de clase completos pueden dar lugar a incrementos considerables (Borzone, 1997; Ehri et al., 2001).

A pesar de las diferencias entre grupos en el nivel global de desempeño, las relaciones entre las habilidades de sensibilidad fonológica y las restantes variables en estudio fueron similares en ambos grupos. Los patrones de correlaciones resultaron similares y los análisis de regresión mostraron el mismo patrón de asociación entre la identificación de rimas y el vocabulario receptivo y entre la detección de sílabas y la tarea de corrección de la pronunciación errónea.

La asociación entre el vocabulario receptivo y la detección de rimas fue encontrada también por otros investigadores. Carrol et al. (2003) encontraron fuertes correlaciones concurrentes entre el conocimiento de vocabulario y la identificación de rimas y sugirieron que esta relación puede ser atribuida al hecho de que la reorganización léxica puede tener efectos iniciales al nivel del ataque y la rima. En coincidencia, Goswami (2002) sostiene que la rima es una unidad fonológica central en la organización de la fonología inglesa.

Ahora bien, la mayor parte de los estudios realizados en inglés en los que se evaluó la identificación de rimas utilizaron como estímulos rimas subsilábicas (rimes), que difieren de las rimas (rhymes) características del español, que constituyen unidades lingüísticas suprasilábicas. En nuestras tareas, los estímulos eran palabras bisílabas graves, en las que la rima se definía como la completa identidad acústica desde la vocal acentuada hasta el último fonema de la palabra. En consecuencia, como sugiere Cardoso-Martins (1995) para el portugués, resulta difícil suponer que en español la rima constituye una unidad fonológica natural. En consecuencia, es poco probable que la reestructuración léxica se produzca a este nivel. Parece más probable que la relación entre el conocimiento de vocabulario y la rima se deba a que, como sugiere Walley (1993), el aumento en el vocabulario incrementa la sensibilidad a la similitud entre sonidos.

En cambio, el apareamiento de sílabas, esto es, la comparación de unidades menores que la rima, puede requerir de representaciones fonológicas más especificadas. Este hecho explicaría la asociación entre la prueba de reconocimiento de sílabas y la tarea de corrección de la pronunciación.

Esta tarea plantea considerables demandas a las representaciones fonológicas de los niños. Los niños deben activar la representación léxica correcta, recuperarla y pronunciarla. Y estos procesos deben tener lugar mientras la pronunciación incorrecta de la palabra puede aún estar activa en la memoria de trabajo del niño, inhibiendo o interfiriendo con el proceso de corrección.

El análisis cualitativo de los errores producidos por los niños al corregir la pronunciación da apoyo a esta interpretación del proceso. En ambos grupos, más del $60 \%$ de las respuestas incorrectas fueron no palabras, con un porcentaje mucho menor de palabras relacionadas semántica o fonológicamente con la palabra intentada. Estas no palabras podían ser una repetición de la pronunciación incorrecta producida por el títere o una no palabra diferente. El primer tipo de error es probablemente resultado de la inhibición de la pronunciación correcta, causada por el input alterado. Es razonable suponer que esta inhibición se produce con mayor frecuencia cuando la representación fonológica de la palabra es débil. Si la representación fonológica de la palabra está bien especificada en el léxico mental del niño, probablemente podrá superar la inhibición. Pero en el caso de representaciones pobremente especificadas, es posible que las pronunciaciones erróneas interfieran en mayor medida. 
El segundo tipo de error - pronunciar una no palabra diferente tanto del input como de la palabra intentada pero fonológicamente similar a ellos - puede ser considerado el resultado de la interacción entre el input y una representación débil. Los resultados obtenidos no nos permiten determinar si la no palabra erróneamente pronunciada por el niño constituye la representación fonológica que el niño tiene de la palabra intentada o si es consecuencia de la inhibición producida por el input alterado. De todos modos, parece razonable suponer que, en cualquier caso, las respuestas erróneas del niño son producto de una representación pobremente especificada en el sentido de que presenta segmentos variables o ausentes que la vuelven más débil y, en consecuencia, más susceptible a la inhibición. Como sostienen Fowler y Swainson (2004), en estas representaciones se ha almacenado alguna información fonológica pero es incompleta, poco clara o difícil de acceder y producir.

Ahora bien, cabe señalar que en ambos grupos la calidad de las representaciones fonológicas estuvo asociada al desempeño en la prueba de reconocimiento de sílabas pero, si bien se encontraron diferencias significativas entre grupos en la prueba de corrección de la pronunciación, no se encontraron diferencias en la prueba de reconocimiento de sílabas. Estos resultados sugieren que, aunque las representaciones precisas parecen relevantes para el procesamiento fonológico, se necesitaría algo más que habilidades de lenguaje oral para el desarrollo de la sensibilidad fonológica, aún a niveles de baja complejidad lingüística, como en el caso de la sílaba (Lonigan et al., 1998; Swan \& Goswami, 1997).

Los resultados obtenidos en este estudio tienen implicancias para la enseñanza. En particular, a pesar de tener un menor conocimiento de vocabulario y, posiblemente, representaciones fonológicas menos especificadas, los niños del grupo de NSE bajo alcanzaron un desempeño similar al de los niños de NSE medio en la prueba de reconocimiento de sílabas. La identificación de sílabas es una habilidad predictora de la adquisición lectora (Jiménez \& Ortiz, 2000). Estos resultados sugieren, entonces, que a pesar de las diferencias en ciertas habilidades básicas, la enseñanza sistemática podría dar a todos los niños las oportunidades de aprendizaje que necesitan para aprender a leer y a escribir.

Desde un punto de vista teórico, estos resultados son convergentes con un creciente cuerpo de evidencia que señala el impacto de la enseñanza sobre el desarrollo lingüístico y cognitivo (Hutzler, Ziegler, Perry, Wimmer \& Zorzi, 2004). Asimismo, señalan la importancia de analizar los contextos educativos como una variable crítica para la configuración de los perfiles cognitivos. 
Anexo

Prueba de reconocimiento de rima

Items de ensayo

\begin{tabular}{lll}
\hline $\begin{array}{l}\text { Pata } \\
\text { Cuna }\end{array}$ & $\begin{array}{l}\text { Lata } \\
\text { Globo } \\
\text { Foco }\end{array}$ & $\begin{array}{l}\text { Carro } \\
\text { Luna } \\
\text { moco }\end{array}$ \\
\hline Ítems de prueba & & \\
\hline moto & foto & \\
tapa & gorro & bolsa \\
gato & plato & mapa \\
lana & rana & tiza \\
pies & mar & dedo \\
sillón & pila & diez \\
papel & cartel & león \\
beso & nave & ñandú \\
Cancha & lancha & queso \\
tela & Nube & baño \\
\hline
\end{tabular}

Prueba de reconocimiento de sílaba

Items de ensayo

\begin{tabular}{lll}
\hline rata & raíz & silla \\
tela & sopa & techo \\
pelo & mano & peso \\
\hline
\end{tabular}

Items de prueba

\begin{tabular}{lll}
\hline cubo & cuchara & llave \\
fideo & fila & camión \\
pato & leche & paraguas \\
melón & mesa & pozo \\
jabón & hilo & jarra \\
Boca & bote & libro \\
nudo & lombriz & nube \\
soda & frasco & sobre \\
cama & caballo & tubo \\
reloj & nido & regla \\
\hline
\end{tabular}


Prueba de corrección de la pronunciación errónea

\section{Items ensayo}

\begin{tabular}{ccc}
\hline Palabra meta & Pronunciación incorrecta del títere & Distractores \\
\hline gato & Kato & perro-vaca \\
mago & Gago & bruja-hada \\
Mesa & Miesa & silla - cama \\
\hline
\end{tabular}

\section{Items de prueba}

\begin{tabular}{lll}
\hline Palabra meta & Pronunciación incorrecta del títere & Distractores \\
\hline lengua & Luenbua & mano-pies \\
copa & Cropa & plato-tenedor \\
fuego & Fego & manguera-balde \\
lana & Blana & hilo-aguja \\
cuello & Quello & brazo-pierna \\
televisión & Tevison & radio-teléfono \\
pantalón & Tantalón & camisa - pollera \\
sombrero & Sobero & corona - bufanda \\
pájaro & Planjaro & nido-gusano \\
Bolsillo & Blolsillo & gorra-moño \\
tractor & Tator & auto-moto \\
globo & Globlo & barrilete-pelota \\
balde & Bande & pala-escoba \\
diente & Dente & boca-nariz \\
jaula & Juaula & bolsa - caja \\
campana & Pampana & carretilla-regadera \\
cocodrilo & Crodilo & elefante-sapo \\
espina & Esplina & gancho - clavo \\
cucaracha & Curacha & araña-abeja \\
Gigante & Gimbante & marciano - robot \\
\hline & &
\end{tabular}




\section{Referencias}

Adams, M. J. (1990). Beginning to read. Thinking and learning about print. Cambridge, Massachusets: MIT Press.

Alameda, J. R., \& Cuetos, F. (1995). Diccionario de frecuencias de las unidades lingüísticas del español. Servicio de Publicaciones de la Universidad de Oviedo.

Araujo, L. (2002). The literacy development of kindergarten English-language learners. Journal of Research in Childhood Education, 16, 232-247.

August, D., \& Shanahan, T. (2006). Developing literacy in second-language learners: Report of the Nacional Reading Panel on language-minority children and youth. Mahwah, NJ: Lawrence Erlbaum Associates.

Borzone, A. M. (1997). El proceso de alfabetización en niños pequeños: diferencias socioculturales. Tesis doctoral no publicada. Universidad de Buenos Aires, Argentina.

Borzone, A. M., \& Diuk, B. (2001). El aprendizaje de la escritura en español: estudio comparativo entre niños de distinta procedencia social. Interdisciplinaria, 18, 35-63.

Borzone, A. M., \& Massone, M.I. (1979). Particularidades acústicas y perceptivas de las vocales españolas. Fonoaudiológica, 25, 94-99.

Borzone, A. M., \& Massone, M.I. (1985).Estrategias de organización fonológica durante el proceso de adquisición del lenguaje, Lenguas Modernas, 12, 149-162.

Bowey, J.A. (1995). Socioeconomic status differences in preschool phonological sensitivity and first-grade reading achievement. Journal of Educational Psychology, 87, 476-487.

Bradley, L., \& Bryant, P. (1983). Categorizing sounds and learning to read: A causal connection. Nature, 301, 419-421.

Bus, A.G., \& van Ijzendoorn, M.H. (1999). Phonological awareness and early reading. A metaanalysis of experimental training programs. Journal of Educational Psychology, 91, 403-414.

Capovilla, A.G.S., \& Capovilla, F.C. (2004). Problemas de Lectura e Escrita. Sao Paulo, SP: Memnon Edicoes Científicas.

Cardoso-Martins, C. (1994). Rhyme perception ¿Global or analytical? Journal of Experimental Child Psychology, 57, 26-41.

Cardoso-Martins, C. (1995). Sensitivity to rhymes, syllables and phonemes in literacy acquisition in Portuguese. Reading Research Quarterly, 30, 808-828.

Carroll, J. M., Snowling, M. J., Hulme, C., \& Stevenson, J. (2003). The development of phonological awareness in preschool children. Developmental Psychology, 5, 913-923.

CEPAL (2009). Panorama social de América Latina 2009. Recuperado el 25 de noviembre de 2009 de http://www.eclac.cl/publicaciones/xml/9/37839/PSE2009-Cap-I-pobreza.pdf

Chaney, C. (1994). Language development, metalinguistic awareness and emergent literacy skills of 3-year-old children in relation to social class. Applied Psycholinguistics, 15, 371-394.

Chatterji, M. (2006). Reading achievement gaps, correlates, and moderators of early reading achievement: Evidence from the Early Childhood Longitudinal Study (ECLS) kindergarten to first grade sample. Journal of Educational Psychology, 98, 489-507.

Chiappe, P., Siegel, L.S., \& Wade-Wooley, L. (2002). Linguistic diversity and the development of reading skills: A longitudinal study. Scientific Studies of Reading, 6, 369-400.

Defior, S., \& Tudela, P. (1994). Effect of phonological training on reading and writing acquisition. Reading and Writing, 6, 299-320. 
Diuk, B. (2007) El aprendizaje inicial de la lectura y la escritura de palabras en español: un estudio de caso. Psykhé, 16, 1, 27-39.

Diuk, B., \& Borzone, A. M. (2006). Las estrategias tempranas de escritura de palabras: análisis del patrón de aprendizaje en niños de distinto sector social de procedencia. Revista IRICE, 19, 1937.

Diuk, B., Signorini, A., \& Borzone, A. M. (2003). Las estrategias tempranas de lectura de palabras en niños de 1er. ciclo de E.G.B.: un estudio comparativo entre niños procedentes de distintos sectores sociales. Psykhé, 12, 2, 51-62.

Dunn, L. M., Lugo, D. E., Padilla, E. R., \& Dunn, L. (1986). Test de Vocabulario en Imágenes Peabody. Circle Pines, MN: AGS Publishing.

Ehri, L. C., Nunes, S. R., Willows, D. M., Schuster, D. V., Yaghoub-Zadeh, Z., \& Shanahan, T. (2001). Phonemic awareness instruction helps children learn to read: Evidence from the National Reading Panel's meta-analysis. Reading Research Quarterly, 36, 250-287.

Elbro, C., Borstrom, I., \& Klint Petersen, D. (1998). Predicting dyslexia from kindergarten: The importance of distinctness of phonological representations of lexical items. Reading Research Quarterly, 33, 36-60.

Ferguson, C. A., \& Farwell, C. (1975). Words and sounds in early language acquisition. Language, $51,491-439$.

Fowler, A. E. (1991). How early phonological development might set the stage for phoneme awareness. En S. Brady \& D.P. Shankweiler (Eds.), Phonological Processes in Literacy (pp. 97-117). Hillsdale, NJ: Erlbaum.

Fowler, A. E. \& Swainson, B. (2004). Relationships of naming skills to reading, memory and receptive vocabulary: Evidence for imprecise phonological representations of words by poor readers. Annals of Dyslexia, 54, 247-280.

Gardner, M. F. (1987). Test figura/palabra del vocabulario expresivo. Buenos Aires: Editorial Médica Panamericana.

Garlock, V. M., Walley, A. C., \& Metsala, J. L. (2001). Age-of-acquisition, word frequency and neighborhood density effects on spoken word recognition: Implications for the development of phoneme awareness and early reading ability. Journal of Memory and Language, 45, 468-492.

Goswami, U. (2002). Phonology, reading development and dyslexia: A cross-linguistic perspective. Annals of Dyslexia, 52, 1-23.

Hecht, S.A., Burgess, S.R., Torgesen, J.K., Wagner, R.K., \& Rashotte, C.A. (2000). Explaining social class differences in growth of reading skills from beginning kindergarten through fourthgrade: The role of phonological awareness, rate of access, and print knowledge. Reading and Writing: An Interdisciplinary Journal, 12, 99-128.

Hecht, S.A., \& Close, L. (2002). Emergent literacy skills and training time uniquely predict variability in response to phonemic awareness training in disadvantaged kindergartners. Journal of Experimental Child Psychology, 82, 93-115.

Hecht, S.A., \& Greenfield, D.B. (2001). Comparing the predictive validity of first-grade teacher ratings and reading related tests on third grade levels of reading skills in young children exposed to poverty. School Psychology Review, 30, 50-59.

Hindson, B., Byrne, B., Fielding-Barnsley, R., Newman, C., Hine, D.W., \& Shankweiler, D. (2005). Assessment and early instruction of preschool children at risk for reading disability. Journal of Educational Psychology, 97, 687-704. 
Hutzler, F., Ziegler, J.C., Perry, C., Wimmer, H., \& Zorzi, M. (2004). Do current connectionist learning models account for reading development in different languages? Cognition, 91, 273296.

Jiménez, J. E., \& Ortiz, M. R. (2000). Conciencia metalingüística y adquisición lectora en la lengua española. The Spanish Journal of Psychology, 3(1), 37-46.

Lonigan, C.J., Burgess, S.R., Anthony, J.L., \& Barker, T.A. (1998). Development of phonological sensitivity in 2- to 5- year-old children. Journal of Educational Psychology, 90, 294-311.

Lonigan, C.J., Burgess, S.R., \& Anthony, J.L. (2000). Development of emergent literacy and early reading skills in preschool children: Evidence from a latent-variable longitudinal study. Developmental Psychology, 36, 596-611.

McBride-Chang, C., Wagner, R.K., \& Chang, L. (1997). Growth modeling of phonological awareness. Journal of Educational Psychology, 89, 621-630.

Metsala, J.L. (1999). Young children's phonological awareness and nonword repetition as a function of vocabulary development. Journal of Educational Psychology, 91, 3-19.

Metsala, J.L., \& Walley, A.C. (1998). Spoken vocabulary growth and the segmental restructuring of lexical representations: Precursors to phonemic awareness and early reading ability. En J.L. Metsala \& J.L.Ehri (Eds.), Word recognition in beginning literacy (pp. 89-119). Mahwah, NJ: Erlbaum.

Muter, V., Hulme, C., Snowling, M.J., \& Stevenson, J. (2004). Phonemes, rhymes, vocabulary and grammatical skills as foundations of early reading development: Evidence from a longitudinal study. Developmental Psychology, 40, 665-681.

Nicholson, T. (1997). Closing the gap on reading failure: social background, phonemic awareness, and learning to read. In B. A. Blachman (Ed.), Foundations of reading acquisition and dyslexia: Implications for early intervention (pp. 381-407). Mahwah, NJ: Erlbaum.

Raz, I.S., \& Bryant, P. (1990). Social background, phonological awareness and children's reading. British Journal of Developmental Psychology, 8, 209-225.

Ricketts, J., Nation, K., \& Bishop, D.V.J. (2007). Vocabulary is important for some, but not all reading skills. Scientific Studies of Reading, 11(3), 235-257

Roberts, T.A. (2005). Articulation accuracy and vocabulary size contributions to phonemic awareness and word reading in English language learners. Journal of Educational Psychology, 97, 601616.

Sautú, R. (1992). Teoría y medición del estatus ocupacional. Escalas ocupacionales objetivas y de prestigio. Cuadernos del Instituto de Investigaciones, Facultad de Ciencias Sociales, Universidad de Buenos Aires, Argentina.

Signorini, A., \& Borzone, A.M. (1996). Las habilidades metafonológicas, la lectura y la escritura en niños de cinco años. Lenguas Modernas, 23, 71-95.

Snow, C.E., Burns, M.S., \& Griffin, P. (1998). Preventing reading difficulties in young children. Washington, DC: National Academy Press.

Swan, D., \& Goswami, U. (1997). Phonological awareness deficits in developmental dyslexia and the phonological representations hypothesis. Journal of Experimental Child Psychology, 66, 18 41.

Thomas, E. M., \& Sénéchal, M. (2004). Long-term effects of articulation on speech perception, phoneme sensitivity, and decoding: A study from age 3 to age 8. Applied Psycholinguistics, 25, 513-541. 
CONOCIMIENTO DE VOCABULARIO, REPRESENTACIONES FONOLÓGICAS Y SENSIBILIDAD FONOLÓGICA EN NIÑOS PEQUEÑOS DE DISTINTO SECTOR SOCIAL DE PROCEDENCIA

Walley, A. (1993) The role of vocabulary development in children's spoken word recognition and segmentation ability. Developmental Review, 13, 286-350.

Walley, A.C., \& Flege, J.E. (1999) Effects of lexical status on the perception of native and non native vowels: A developmental study. Journal of Phonetics, 27, 307-32

Wesseling, R., \& Reitsma, P. (2001). Preschool phonological representations and development of reading skills. Annals of Dyslexia, 52, 203-229. 
